\title{
A Machine Learning Algorithm for Biomedical Images Compression Using Orthogonal Transforms
}

\author{
Aurelle Tchagna Kouanou \\ Research Unity of Condensed Matter, Electronics and Signal Processing, Department of Physics, Faculty of Science, \\ University of Dschang, P.O.Box 67 Dschang, Cameroon. \\ Email: tkaurelle@gmail.com \\ Daniel Tchiotsop \\ Unité de Recherche d'Automatique et d'Informatique Appliquée (LAIA), IUT-FV de Bandjoun, Université de \\ Dschang-Cameroun, B.P. 134 Bandjoun. \\ Email: daniel.tchiotsop@gmail.com \\ René Tchinda \\ Unité de Recherche d'Ingénierie des Systèmes Industriels et de l'Environnement (LISIE), IUT-FV de Bandjoun, \\ Université de Dschang- Cameroun, B.P. 134 Bandjoun. \\ Email: ttchinda@yahoo.fr \\ Christian Tchito Tchapga, Adelaide Nicole Kengnou Telem and Romanic Kengne \\ Research Unity of Condensed Matter, Electronics and Signal Processing, Department of Physics, Faculty of Science, \\ University of Dschang, P.O.Box 67 Dschang, Cameroon. \\ Email: tchitochristian@gmail.com, adelkengnou@yahoo.com, kengneromanic@gmail.com
}

Received: 07 February 2018; Accepted: 13 August 2018; Published: 08 November 2018

\begin{abstract}
Compression methods are increasingly used for medical images for efficient transmission and reduction of storage space. In this work, we proposed a compression scheme for colored biomedical image based on vector quantization and orthogonal transforms. The vector quantization relies on machine learning algorithm (K-Means and Splitting Method). Discrete Walsh Transform (DWaT) and Discrete Chebyshev Transform (DChT) are two orthogonal transforms considered. In a first step, the image is decomposed into sub-blocks, on each sub-block we applied the orthogonal transforms. Machine learning algorithm is used to calculate the centers of clusters and generates the codebook that is used for vector quantization on the transformed image. Huffman encoding is applied to the index resulting from the vector quantization. Parameters Such as Mean Square Error (MSE), Mean Average Error (MAE), PSNR (Peak Signal to Noise Ratio), compression ratio, compression and decompression time are analyzed. We observed that the proposed method achieves excellent performance in image quality with a reduction in storage space. Using the proposed method, we obtained a compression ratio greater than 99.50 percent. For some codebook size, we obtained a MSE and MAE equal to zero. A comparison between DWaT, DChT method and existing literature method is performed. The proposed method is really appropriate for biomedical images which cannot tolerate
\end{abstract}

distortions of the reconstructed image because the slightest information on the image is important for diagnosis.

Index Terms-Biomedical Color Image, Machine Learning, Vector Quantization, Discrete Walsh Transform, Discrete Chebyshev Transform.

\section{INTRODUCTION}

Biomedical image compression is an extremely important part of modern computing. By having the ability to compress images from their original size, the required storage space in computer memory space of images can be reduced. In addition, the transmission becomes easier and less time-consuming. In fact, the problem is the very large amount of data contained in a medical image can quickly saturate conventional systems [1]. There exist two types of compression: lossless compression where reconstruction data is identical to the original; and lossy compression where there is a loss of data. However, lossless compression is limited because compression rates are very low [2]. The compression ratio of the lossy compression scheme is very high. Lossy compression algorithms are generally based on orthogonal processes, such as the discrete cosine transform [3-6], the discrete Walsh transform [3], the 
Karhunen-Loeve transform [3, 5, 7], the discrete wavelet transform [1, 2, 4, 5, 8, 9] and the Discrete Chebyshev Transform (DChT) [10-13]. All these transforms are unitary, symmetrical, reversible and the energy of the image before and after processing remains unchanged. However, although these compression methods can have a good image quality of restoration with high compression rates, they are slow in terms of coding time and do not produce a good compromise between the Peak Signal to Noise Ratio (PSNR) and the compression ratio (CR). To overcome this drawback, vector quantization (VQ) and Machine Learning (ML) algorithms are most often associated to increase the performance. VQ techniques have been widely used in the area of image coding because of their ability to achieve a low bit rate [14-19]. VQ techniques achieve a low bit rate by exploiting the correlation and redundancy between blocks [20, 21]. VQ breaks the transformed image to be compressed into vectors which are quantized to the closest codeword. VQ is achieved by only sorting or transmitting the address of the closest codeword, instead of the whole vector of image pixels [22]. To improve the VQ, a ML algorithm can be used to calculate the codebook. Also, $K$-Means algorithm is often used to calculate and generate the optimal codebook that can be adapted to the transformed image [23, 24]. Several papers have been published concerning image compression using the orthogonal transform and some analytical solutions are described in [2-5]. Lu and Chang presented in [15] a snapshot of the recently developed schemes. The discussed schemes include mean distance ordered partial codebook search (MPS), enhance LBG (Linde, Buzo and Gray) and some intelligent algorithms. In [12], Prattipati and al compare integer Cosine and Chebyshev transform for image compression using variable quantization. The aims of their work were to examine cases where DChT could be an alternative compression transform to DCT. However, their work was limited to the grayscale image compression and further, the use of a scalar quantification does not allow them to reach a high PSNR. H. B. Kekre, P. Natu and T. Sarode presented in
[19] a fusion of Vector Quantization and Hybrid Wavelet Transform (HWT). They proposed a color image compression method based on two types of vector quantization: Kekre's Median Codebook Generation (KMCG) and Kekre's Fast Codebook Generation (KFCG). They obtained fewer errors than in the LBG algorithm and the image quality is better.

For biomedical images, all the information may be needed for diagnosis. Diagnosis based on the biomedical image cannot tolerate the distortion on the image. After the compression process, the reconstructed image should be identical to the original image. To achieve high rates, we focused on the two orthogonal transforms (Walsh and Chebyshev). We adapt these methods by an intelligent algorithm for high compression rate and no loss of data. Our method minimizes the distortions on the reconstructed images. Further, we observed that when the image is transformed with DWaT or DChT, and the codebook is calculated with a learning algorithm, the compression has very good parameters.

The rest of the paper is as below. Section 2 briefs on the concept of discrete Walsh transform, discrete Chebyshev transform and describes both vector quantization and Huffman encoding. Section 3 includes the results and the performance analysis. The implementation issues and comparisons are performed. Section 4 concludes the work.

\section{Materials AND Methods}

In this session, we describe the set of methods used to develop our compression algorithm. In our proposed algorithm, we use a Machine Learning algorithm. $K$ Means clustering is an unsupervised learning algorithm that helps us with the Split method to construct our codebook necessary for vector quantization step. Thus, in this session, we will describe the three main steps of our compression scheme. The Fig. 1 illustrates the block diagram of the proposed compression and decompression scheme.

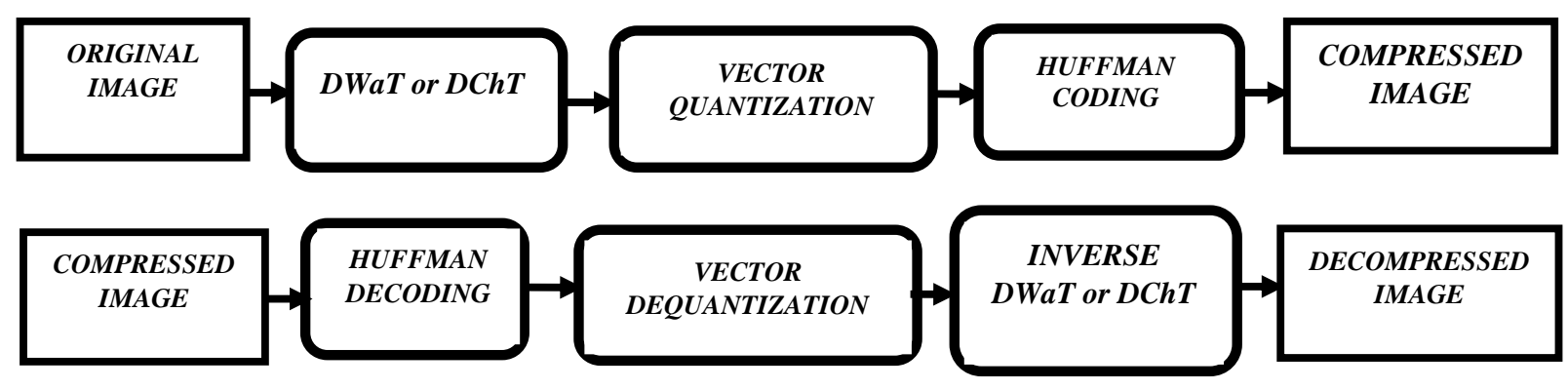

Fig.1. Block diagram of compression and decompression scheme. 
The transformations refer to the conversion of the images from the time domain to other domains such as frequency domain through orthogonal bases. The image in the new domain brings out specific innate characteristics which can be suitable for some applications. In this work, we used Walsh transform and Chebyshev transform.

\section{A. Discrete chebyshev transform (dcht)}

Chebyshev transform is obtained from the Chebyshev polynomials. The orthogonal Chebyshev polynomials are commonly used to analyze, approximate, synthesize, or reconstruct signals and images [25]. Discrete Chebyshev Transform (DChT) is an approach based on discrete Chebyshev polynomials. For a given positive integer $N$ (the vector size) and a value $n$ in the range $[1, N-1]$, the $N$ order orthogonal Chebyshev polynomials $T_{k}(x), k=1,2, \ldots, N-1$ are defined using the following recursive relation defined in [10-12, 26-28]:

$$
\begin{gathered}
T_{0}(x)=\frac{1}{\sqrt{N}}, \\
T_{k}(0)=\sqrt{\frac{N-k}{N+k} \sqrt{\frac{2 k+1}{2 k-1}} T_{k-1}(0),} \\
T_{k}(1)=\left\{1+\frac{k(k+1)}{1-N}\right\} T_{k}(0), \\
T_{k}(x)=\gamma_{1} T_{k}(x-1)+\gamma_{2} T_{k}(x-2), \\
k=1,2, \ldots, N-1, x=2,3, \ldots,\left(\frac{N}{2}-1\right),
\end{gathered}
$$

Where

$$
\begin{gathered}
\gamma_{1}=\frac{-k(k+1)-(2 x-1)(x-N-1)-x}{x(N-x)}, \\
\gamma_{2}=\frac{(x+1)(x-N-1)}{x(N-x)},
\end{gathered}
$$

The forward DChT of order $N$ is defined in [12, 27-29] by (8):

$$
F(k)=\sum_{x=0}^{N-1} f(x) T_{k}(x)
$$

$k=0,1,2, \ldots, N-1$

Where $F(k)$ denotes the coefficient of orthonormal Chebyshev polynomials. $f(x)$ is a one-dimension signal at time index $\mathrm{x}$. The inverse DChT is given in [27-29] by (9):

$$
f(x)=\sum_{k=0}^{N-1} F(k) T_{k}(x)
$$

$x=0,1,2, \ldots, N-1$

Now, let consider a function with two variables $f(x, y)$ sampled on a square grid $N x N$ points. Its DChT is given by (10), [12, 29-31]:

$$
F(j, k)=\sum_{x=0}^{N-1} \sum_{y=0}^{N-1} f(x, y) T_{j}(y) T_{k}(x)
$$

$j, k=0,1,2, \ldots, N-1$

And its inverse DChT is given by (19):

$$
f(x, y)=\sum_{j=0}^{N-1} \sum_{k=0}^{N-1} F(j, k) T_{j}(y) T_{k}(x)
$$

$x, y=0,1,2, \ldots, N-1$

\section{B. Discrete walsh transform (dwat)}

Walsh functions are already discrete and thus easy to manipulate. The sequence of a Walsh function is determined as the number of zero-crossings per unit time interval.

The Walsh function $W(k, x)$ is defined on the interval [0 1] in [32] by (12)

$$
W(k, x)=\sum_{n=0}^{2^{\gamma}-1} W_{k}\left(n / 2^{k}\right) \operatorname{win}\left(x 2^{\gamma}, n, n+1\right)
$$

where $N=2^{k}$;

$$
\operatorname{win}(x, a, b)= \begin{cases}1 & \text { for } x \in(a, b) \\ 0 & \text { for } x \notin[a, b] \\ \frac{1}{2} & \text { for } x \in\{a, b\}\end{cases}
$$

win represented the window function

When $x=m / N$ and for some integer $0<m<N$ it comes that

$W(k, m / N)=W_{k}(m / N) \delta_{m, n}=W_{k}(n / N) \delta_{m, n}=W_{k}(m, k / N)$

The orthonormality property of the Walsh functions is expressed by (14) 


$$
(W(m, x), W(n, x))=\delta_{m, n}
$$

The discrete orthogonality of Walsh functions is defined in [32] by (15)

$$
\sum_{i=0}^{N-1} W(m, i / N) W(n, i / N)=N \delta_{m, n}
$$

Consider a function $f(n)$ defined in a discrete form on a compact, its Walsh transform is given in [32, 33] by (16)

$$
F(m)=\sum_{n=0}^{N-1} f(n) W_{k}(m, n)
$$

Where $W_{k}(m, n)$ is a Walsh function of rank $m$, with $m=0,1,2, \ldots \ldots \ldots, N-1$

The inverse DWaT is defined in [33] by (17):

$$
f(n)=\frac{1}{N} \sum_{m=0}^{N-1} F(m) W_{k}(m, n)
$$

Where with $n=0,1,2, \ldots, N-1$

For a function with two variables $f(n, m)$ sampled on square grid $N^{2}$ points, its $2 \mathrm{D}$ Walsh transform is given in [34] by (18)

$$
F(u, v)=\sum_{n=0}^{N-1} \sum_{n=0}^{N-1} W_{k}(u, v) f(n, m) W_{k}(u, v)
$$

Where $W_{k}(u, v)$ is the Walsh matrix having the same size as the function $f(n, m)$. The inverse 2D transform is also defined in [34] by (19)

$$
f(n, m)=\frac{1}{N^{2}} \sum_{n=0}^{N-1} \sum_{n=0}^{N-1} W_{k}(n, m) F(u, v) W_{k}(n, m)
$$

Two techniques are used to perform the quantization of coefficients resulting from the discrete Walsh transform and discrete Chebyshev transforms, scalar quantization and vector quantization. If one refers to the theory of the distortion factor introduced by Shannon [2, 4], the best performance is always reached in theory with vectors instead of scalars. We have then adopted vector quantization of DWaT and DChT coefficients.

\section{Vector quantization \\ a. Basic concepts}

Vector quantization (VQ) was developed by Gersho and Gray [16, 17, 35]. A vector quantizer $q$ may be defined in [36] by an application of a set $E$ into a set $F \subset E$ as (20).

$$
\begin{gathered}
E \rightarrow F \subset E \\
x=\left(x_{0}, \ldots, x_{k-1}\right) \rightarrow q(x) \\
\hat{\imath} \hat{x} \in \hat{A}=\left\{\begin{array}{l}
\left.y_{i}, i=1, \ldots, M\right\}, \\
y_{i} \in F
\end{array}\right.
\end{gathered}
$$

$\hat{A}$ is a dictionary of size $M$ and dimension $k . E$ is partitioned by $S=\left\{S_{i}, i=1, \ldots, M\right\}$, with $S_{i}=\left\{\frac{x}{q(x)}=y_{i}\right\}$. The distortion between $\hat{x}$ and $x d(\hat{x}, x)$ is a positive quantity or zero [18-21, 35-42] given by (21):

$$
d(\hat{x}, x)=\|X-\hat{X}\|^{2}=\sum_{i=1}^{L}(X(i)-\hat{X}(i))^{2}
$$

Where $\mathrm{L}$ is the vector size.

The total distortion of the quantizer for a given codebook is then:

$$
D(q)=\left(\frac{1}{L}\right) \times \sum_{i=1}^{L}\left(\underset{Y_{j} \in \text { Codebook }}{\operatorname{Minimum}} d\left(X_{i}, Y_{j}\right)\right)
$$

The optimal quantizer $\left(q^{*}\right)$ is that which minimizes the distortion for a random sequence of vectors $X$, where $X$ has the probability $p(X)$, it has to verify the condition $D\left(q^{*}\right) \leq D(q) . \hat{A}$ and $S$ determine entirely $q$ and we can write :

$$
D(q)=D(\hat{A}, S)
$$

Where

$$
\begin{aligned}
& D(\hat{A}, S)=E(d(X, q(X))) \\
& =\sum_{i}^{N} P_{r}\left(x \in S_{i}\right) \int d\left(x, y_{i} / x \in S_{i}\right) p\left(x / x \in S_{i}\right) d x
\end{aligned}
$$

Most of the classical vector quantization algorithms are based on two important properties [36, 43]:

- If $\hat{A}$ is given, then the best partition of the input space is $p(\hat{A})$, obtained by matching each $X$ to vector $y_{i}$ to $\hat{A}$ minimizing $d\left(x, x_{j}\right)$. This rule of nearest neighbor.

- $\quad$ If $S$ is given, then assume that for every $S$ nonzero probability in a space k-Euclidean,

$$
\begin{aligned}
& \exists \hat{x}(S) / E(d(X, \hat{x}(S)) / X \in S) \\
& =\min _{u} E(d(X, u) / X \in S)
\end{aligned}
$$


$\hat{x}(S)$ thus corresponds to a generalized centroid to $S$. Under these conditions, the best alphabet to reproduction is $\hat{x}(S)=\left\{\hat{x}\left(S_{i}\right), i=1, \ldots, N\right\}$.

For the construction of our codebook, we used a machine learning algorithm that performs with unsupervised learning. Our unsupervised learning relies on the $K$-Means algorithm with the splitting method.

\section{b. K-Means algorithm}

The K-Means algorithm is at the basis of many standard methods of vector quantization [23]. Proceeding from $M$ training vectors $x(n), 1 \leq n \leq M$, the problem is to split this set into $K$ groups $S_{i}$ such that the optimal conditions are satisfied. The procedure of data classification is simple and easy for clustering [23, 44, 45]. K-Means is an algorithm leading to a local optimum satisfying the following conditions [46, 47]:

a) Choose $K$ vectors $Y_{i}(0)$, initialize $\mathrm{m}$, the index of iterations, to 0 ;

b) Classification: at iteration $\mathrm{m}$, store the vectors $\{x(n), n=1, \ldots, M\}$ in the $S_{i}(m)$ by applying the rule of the nearest neighbor from the vectors $Y_{j}(m)$;

c) Updating of code vectors $Y_{j}(m+1)=$ $\operatorname{centroid}\left(S_{i}(m)\right), 1 \leq i \leq K$;

d) End of the test on the gain in distortion compared to the previous iteration. If the result is negative, go to the step b.

It is obvious that in $K$-Means, we need all the training sequence for every iteration and the quantizer is not available as long as the procedure is not completed. Thus, the data must be part of the training sequence, and the groups change with each iteration. This is ideal for the case with medical images in which the images that represent data have to be used for the construction of codebooks. This is not the case of some method such as the most use in vector quantization Linde, Buzo and Gray (LBG) algorithm $[15,35,48]$. In the LBG algorithm, a part only of the input sequence is used to construct the quantizer, and, provided that the input sequence of vectors produced by the source is stationary and ergodic, the implementation remains practically optimal for any vector produced later. Since we are manipulating medical images, we chose for our compression scheme the $K$ Means algorithm instead of using a part of the input sequence to build the quantizer. We used all the input sequence in order to have a very low distortion. The algorithm of $K$-Means in our scheme is used with the splitting method. Vectors have the same probability and the entropy $\mathrm{H}$ increases.

\section{c. Splitting Method}

The Splitting operation serves to modify the initial codebook used by the $K$-Means algorithm. It is based on the probabilities of occurrence $P_{i}$ of vectors of the codebook obtained during the first classification. A vector $i$ of the codebook is then split if its probability $P_{i}$ is high. Thus, the splitting of vectors of the codebook helps to balance the distribution of vectors of the training sequence during classification and thus, optimizes the partition of the codebook. Indeed, a splitting is successful if a class $S^{i}$ containing a $V_{i}$ vector is split in two class $V_{i} / 2$ vectors: one vector that has a great probability of occurrence is then replaced by two equiprobably vectors of lower probability of occurrence $[49,50]$.

VQ makes it possible to have a good quantification of the image during the compression. However the codebook generated during quantification is not always optimal, the reason why we introduced a training algorithm ( $K$-Means) to calculate the codebook. $K$-Means calculate and generate the optimal codebook that can be adapted to the transformed image [23]. $K$-Means does not solve the equiprobability problem between the vectors. Thus we coupled the $K$-Means with the Split method to have equiprobably vectors of lower probability of occurrence. This allows us to create a very suitable codebook for image compression and to be able to close the lossless compression techniques.

\section{Huffman coding}

The Huffman coding is a compression algorithm based on the frequencies appearance of characters in an original document. Developed by David Huffman in 1952, the method relies on the principle of allocation of shorter codes for frequent values and longer codes to less frequent values [2, 4, 51-54]. This coding uses a table containing the appearance frequencies of each character to establish an optimal binary string representation. The procedure is decomposed into three parts:

- First, the creation of the frequencies appearance of the character table in the original data.

- Afterwards, the creation of a binary tree according to the previously calculated table.

- And lastly, encoding symbols in an optimal binary representation.

\section{E. Performance evaluation}

There are four parameters to evaluate the performance of our compression scheme: Mean Square Error (MSE), Maximum Absolute Error (MAE), Peak Signal to Noise Ratio (PSNR) and the Compression rate (CR). For an image represented by 256 grayscale ranging between 0 and 255. The MSE and PSNR are defined in [14, 21] as (25).

$$
M S E=\frac{1}{M^{*} N} \sum_{i=0}^{M-1} \sum_{j=0}^{N-1}\left(I_{0}(i, j)-I_{r}(i, j)\right)^{2}
$$

With $I_{0}$ image as to compress and $I_{r}$ reconstructed image and $(M, N)$ the size of the image. The PSNR is given by (26).

$$
P S N R=10 \log _{10}\left(\frac{255^{2}}{M S E}\right)
$$


The MSE represents the mean error into energy. It indicates the energy of the difference between the original image and the reconstructed image. PSNR gives an objective measure of the distortion introduced by the compression. It is expressed in deciBels $(\mathrm{dB})$. For a high PNSR and a low MSE, the decompressed image is closed to the original image.

The Compression rate (CR) is defined as the ratio between the uncompressed size and compressed size. The $\mathrm{CR}$ is given in $[2,4,55]$ by $(27)$ and $(28)$.

$$
C R=\frac{\text { size of compressed image }}{\text { size of original image }}
$$

In percentage from the CR expression is given by (28)

$$
C R(\%)=100-\frac{\text { size of compressed image }}{\text { size of original image }} X 100
$$

Maximum Absolute Error (MAE) defined by (29) shows the worst-case error occurring in the compressed image [56].
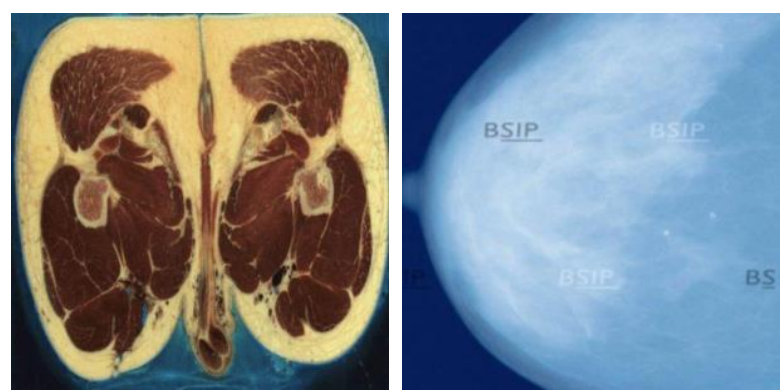

(a) Pelvis- (I1)

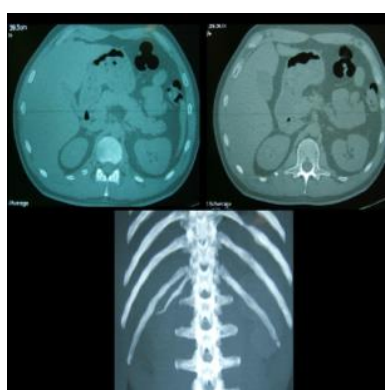

(b) Mammography- (I2)

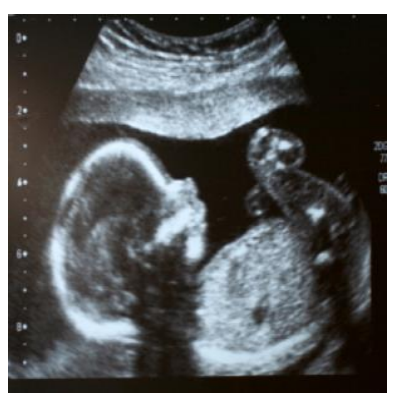

(e) Fracture- (I5) (f) Echography- (I6)

$$
M A E=\max \left|I_{0}(i, j)-I_{r}(i, j)\right|
$$

\section{RESUlTS AND DiSCUSSION}

In order to validate our proposed algorithm for biomedical images compression, we have performed an objective evaluation with the parameters presented in the previous session on test images from Fig.2. Thus, we present our obtained results with two utilized transforms: DWaT, DChT. The comparison between DWaT and DChT are made. We use our algorithms on the images of other articles finally to compare our algorithm with those of the literature. This in order to conclude on the effectiveness of our algorithms.

\section{A. Results}

MATLAB software was used to simulate our new image compression scheme. We use eight images from $[57,58]$ as test images to evaluate our scheme compression. These images represented in the fig. 2 . Image size is $512 \times 512 \times 3$ bytes.

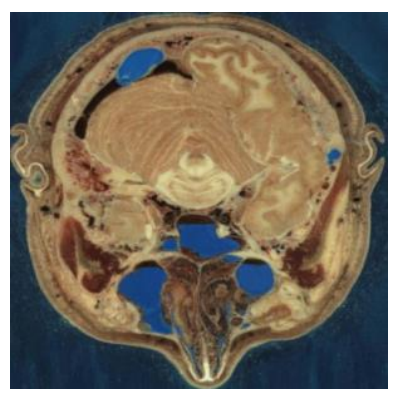

(c) Head - (I3)

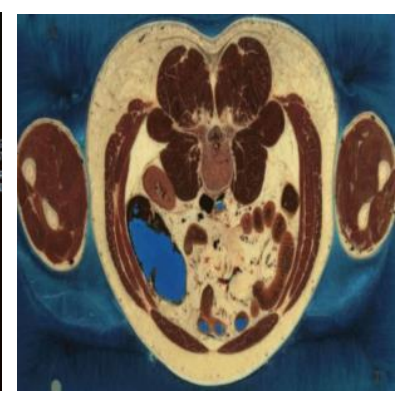

(g) Abdomen- (I7)

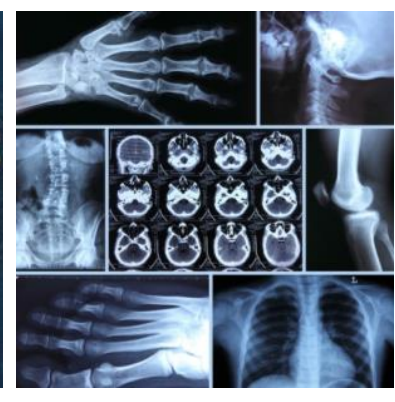

(d) General- (I4)

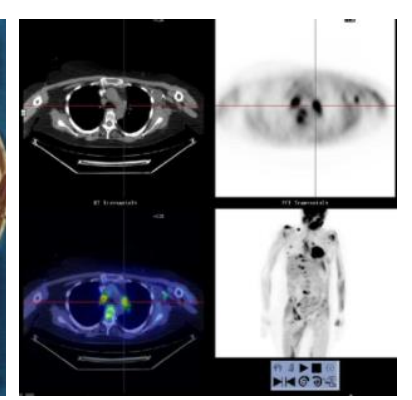

(h) Strange Body- (I8)

Fig.2. The test images $[57,58]$

Table 1 presents the results obtained with DChT and table 2 those obtained with DWaT. 
Table 1. Recapitulative of results with DChT Method

\begin{tabular}{|c|c|c|c|c|c|}
\hline \multirow{2}{*}{ Images } & \multirow{2}{*}{$\begin{array}{l}\text { Evaluation } \\
\text { Parameters }\end{array}$} & \multicolumn{4}{|c|}{ Codebook Size } \\
\hline & & 64 & 128 & 256 & 512 \\
\hline \multirow{6}{*}{ Pelvis- (I1) } & PSNR (dB) & 20.85 & 24.38 & 33.35 & Inf \\
\hline & MSE & 534.11 & 236.98 & 30.01 & 0 \\
\hline & MAE & 7.78 & 4.94 & 1.76 & 0 \\
\hline & CR $(\%)$ & 99.90 & 99.87 & 99.82 & 99.78 \\
\hline & CT (s) & 175.81 & 172.57 & 175.44 & 201.64 \\
\hline & DT (s) & 12055.1 & 10943.55 & 12654.48 & 12319.96 \\
\hline \multirow{6}{*}{ Mammography- (I2) } & PSNR (dB) & 34.55 & 40.64 & 50.46 & Inf \\
\hline & MSE & 22.77 & 5.60 & 0.58 & 0 \\
\hline & MAE & 1.41 & 0.72 & 0.18 & 0 \\
\hline & CR (\%) & 99.90 & 99.86 & 99.82 & 99.78 \\
\hline & CT (s) & 180.65 & 175.76 & 178.53 & 191.56 \\
\hline & DT (s) & 12698.6 & 11075.48 & 11111.27 & 11123.04 \\
\hline \multirow{6}{*}{ Head- (I3) } & PSNR (dB) & 23.14 & 26.82 & 37.05 & Inf \\
\hline & MSE & 315.16 & 135.03 & 12.81 & 0 \\
\hline & MAE & 5.98 & 3.67 & 1.19 & 0 \\
\hline & CR (\%) & 99.91 & 99.87 & 99.82 & 99.78 \\
\hline & CT (s) & 173.69 & 169.75 & 178.62 & 198.76 \\
\hline & DT (s) & 10746.27 & 11016.27 & 10705.48 & 11614.41 \\
\hline \multirow{6}{*}{ General- (I4) } & PSNR (dB) & 18.41 & 21.42 & 27.73 & Inf \\
\hline & MSE & 936.89 & 468.14 & 109.54 & 0 \\
\hline & MAE & 9.93 & 6.40 & 2.83 & 0 \\
\hline & CR (\%) & 99.89 & 99.86 & 99.82 & 99.78 \\
\hline & CT (s) & 167.15 & 164.41 & 207.24 & 194.43 \\
\hline & DT (s) & 11770.12 & 11528.36 & 10794.08 & 12188.45 \\
\hline \multirow{6}{*}{ Fracture- (I5) } & PSNR (dB) & 21.89 & 24.70 & 32.61 & 71.80 \\
\hline & MSE & 419.87 & 220.33 & 35.60 & 0.004 \\
\hline & MAE & 5.72 & 3.80 & 1.28 & 0.00008 \\
\hline & CR (\%) & 99.89 & 99.85 & 99.82 & 99.78 \\
\hline & $\mathrm{CT}(\mathrm{s})$ & 164.62 & 165.10 & 184.36 & 241.83 \\
\hline & DT (s) & 10468.87 & 10774.21 & 10762.52 & 11022.65 \\
\hline \multirow{6}{*}{ Echography- (I6) } & PSNR (dB) & 23.88 & 29.09 & 36.95 & 56.09 \\
\hline & MSE & 265.75 & 80.01 & 13.11 & 0.15 \\
\hline & MAE & 4.90 & 2.55 & 1.16 & 0.06 \\
\hline & CR $(\%)$ & 99.87 & 99.82 & 99.76 & 99.70 \\
\hline & $\mathrm{CT}(\mathrm{s})$ & 146.23 & 155.49 & 170.2 & 199.29 \\
\hline & DT (s) & 8945.45 & 10076.84 & 10308.12 & 10767.65 \\
\hline \multirow{6}{*}{ Abdomen- (I7) } & PSNR (dB) & 21.11 & 24.91 & 33.28 & Inf \\
\hline & MSE & 502.9 & 209.49 & 30.54 & 0 \\
\hline & MAE & 7.30 & 4.47 & 1.80 & 0 \\
\hline & CR (\%) & 99.89 & 99.87 & 99.82 & 99.78 \\
\hline & $\mathrm{CT}(\mathrm{s})$ & 160.2 & 168.71 & 218.65 & 276.15 \\
\hline & DT (s) & 10301.2 & 10564.08 & 11187.09 & 11279.2 \\
\hline \multirow{6}{*}{ Strange Body- (I8) } & PSNR (dB) & 23.34 & 26.76 & 34.37 & Inf \\
\hline & MSE & 301.13 & 137.10 & 23.73 & 0 \\
\hline & MAE & 4.04 & 2.51 & 0.86 & 0 \\
\hline & CR (\%) & 99.90 & 99.87 & 99.82 & 99.78 \\
\hline & CT (s) & 172.71 & 165.91 & 176.24 & 216.17 \\
\hline & DT (s) & 12868.87 & 12390.25 & 12902.74 & 13011.2 \\
\hline
\end{tabular}


Table 2. Recapitulative of results with DWaT Method

\begin{tabular}{|c|c|c|c|c|c|}
\hline \multirow{2}{*}{ Images } & \multirow{2}{*}{$\begin{array}{l}\text { Evaluation } \\
\text { Parameters }\end{array}$} & \multicolumn{4}{|c|}{ Codebook Size } \\
\hline & & 64 & 128 & 256 & 512 \\
\hline \multirow{6}{*}{ Pelvis- (I1) } & PSNR (dB) & 21.05 & 24.28 & 31.15 & Inf \\
\hline & MSE & 510.11 & 242.54 & 49.88 & 0 \\
\hline & MAE & 7.70 & 5.08 & 2.42 & 0 \\
\hline & CR (\%) & 99.89 & 99.86 & 99.82 & 99.78 \\
\hline & $\mathrm{CT}(\mathrm{s})$ & 6.93 & 9.89 & 16.06 & 34.27 \\
\hline & DT (s) & 11.43 & 14.99 & 22.22 & 43.57 \\
\hline \multirow{6}{*}{ Mammography- (I2) } & PSNR (dB) & 35.33 & 41.35 & 50.01 & Inf \\
\hline & MSE & 19.05 & 4.75 & 0.64 & 0 \\
\hline & MAE & 1.31 & 0.68 & 0.21 & 0 \\
\hline & CR (\%) & 99.85 & 99.80 & 99.75 & 99.78 \\
\hline & CT (s) & 4.95 & 5.99 & 9.59 & 32.09 \\
\hline & DT (s) & 7.88 & 9.30 & 14.16 & 40.93 \\
\hline \multirow{6}{*}{ Head- (I3) } & PSNR (dB) & 23.06 & 26.33 & 33.52 & Inf \\
\hline & MSE & 321.0 & 151.29 & 28.89 & 0 \\
\hline & MAE & 6.07 & 4.02 & 1.76 & 0 \\
\hline & CR (\%) & 99.90 & 99.86 & 99.82 & 99.78 \\
\hline & $\mathrm{CT}(\mathrm{s})$ & 7.08 & 9.54 & 16.72 & 36.52 \\
\hline & DT (s) & 11.97 & 14.38 & 22.93 & 46.15 \\
\hline \multirow{6}{*}{ General- (I4) } & PSNR (dB) & 19.21 & 22.86 & 27.57 & Inf \\
\hline & MSE & 780.1 & 336.2 & 113.8 & 0 \\
\hline & MAE & 9.08 & 5.50 & 3.14 & 0 \\
\hline & CR (\%) & 99.89 & 99.85 & 99.81 & 99.78 \\
\hline & CT (s) & 6.67 & 9.86 & 16.18 & 31.02 \\
\hline & DT (s) & 11.38 & 14.76 & 22.72 & 42.91 \\
\hline \multirow{6}{*}{ Fracture- (I5) } & PSNR (dB) & 21.78 & 24.20 & 28.14 & 51.39 \\
\hline & MSE & 430.67 & 246.85 & 99.6 & 0.47 \\
\hline & MAE & 5.79 & 4.14 & 2.45 & 0.02 \\
\hline & CR (\%) & 99.88 & 99.85 & 99.81 & 99.78 \\
\hline & CT (s) & 6.95 & 9.04 & 13.74 & 69.16 \\
\hline & DT (s) & 11.50 & 13.97 & 19.84 & 78.01 \\
\hline \multirow{6}{*}{ Echography- (I6) } & PSNR (dB) & 23.89 & 28.73 & 36.95 & 50.38 \\
\hline & MSE & 265.78 & 87.04 & 13.11 & 0.59 \\
\hline & MAE & 4.95 & 2.70 & 1.16 & 0.19 \\
\hline & CR (\%) & 99.87 & 99.82 & 99.76 & 99.70 \\
\hline & $\mathrm{CT}(\mathrm{s})$ & 5.29 & 7.70 & 12.15 & 23.98 \\
\hline & DT (s) & 8.87 & 11.78 & 17.44 & 32.57 \\
\hline \multirow{6}{*}{ Abdomen- (I7) } & PSNR (dB) & 21.11 & 24.60 & 30.93 & Inf \\
\hline & MSE & 502.9 & 225.3 & 52.45 & 0 \\
\hline & MAE & 7.30 & 4.69 & 2.36 & 0 \\
\hline & CR (\%) & 99.89 & 99.86 & 99.82 & 99.78 \\
\hline & $\mathrm{CT}(\mathrm{s})$ & 7.04 & 9.78 & 16.71 & 35.48 \\
\hline & DT (s) & 11.48 & 14.77 & 22.53 & 44.58 \\
\hline \multirow{6}{*}{ Strange Body- (I8) } & PSNR (dB) & 23.40 & 26.03 & 31.80 & 52.06 \\
\hline & MSE & 297.6 & 162.17 & 42.95 & 0.40 \\
\hline & MAE & 3.79 & 2.55 & 1.79 & 0.02 \\
\hline & CR (\%) & 99.89 & 99.85 & 99.82 & 99.78 \\
\hline & $\mathrm{CT}(\mathrm{s})$ & 7.62 & 11.16 & 15.35 & 72.45 \\
\hline & DT (s) & 12.24 & 16.26 & 21.18 & 81.48 \\
\hline
\end{tabular}


We can observe in Table. 1 and in Table. 2 the variation of PSNR, MSE, MAE, CR, CT (Compression Time) and DT (Decompression Time) according to codebook size. For a codebook size of 512, the values of PSNR are very high and they closer infinity for some images. The compression ratio ranges between $99.72 \%$ and $99.90 \%$ whatever the image and the codebook size. We can also notice that when the codebook size increases, the CR decreases slightly. Our results are then very satisfactory given the good compromise between PSNR and CR. The Fig. 3 and Fig. 4 present some of our test images decompressed using DChT and DWaT respectively. From these images, we confirm that the quantization step enormously influences the compression system especially as regards the choice of codebook size. For the same simulation with the same configurations, the settings such as PNSR, MSE and CR may be slightly different. This is because we assumed in our program during the learning phase, that if no vector is close to the codeword, we use a random vector by default. We can also see that the image quality is good when the codebook size is 256 or 512 .

Head- (I3)

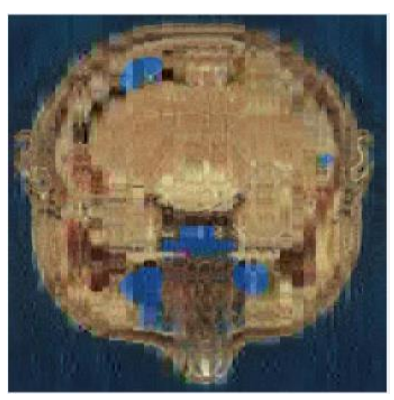

Codebook Size: 64

PNSR: 23.14dB; MAE: 5.98;

MSE: 315.16; CR: 99.91\%;

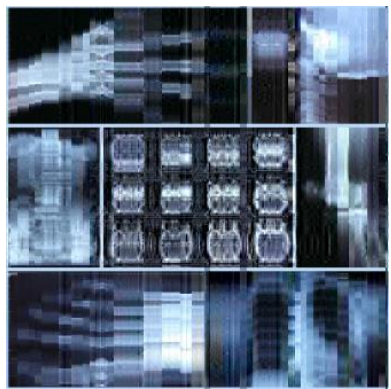

Codebook Size: 64

PNSR: 18.41dB; MAE: 9.93;

MSE: 936.89; CR: 99.89\%;

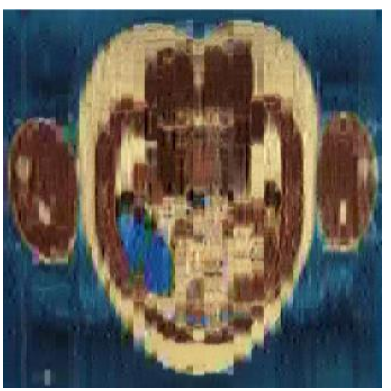

Codebook Size: 64

PNSR: 21.11dB; MAE: 7.30;

MSE: 502.9; CR: 99.89\%;

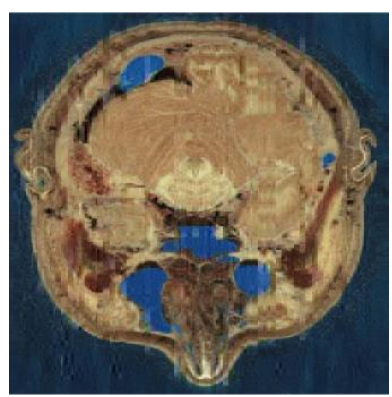

Codebook Size: 128

PNSR: 26.82dB; MAE: 3.67;

MSE: 135.03; CR: 99.87\%;

General-

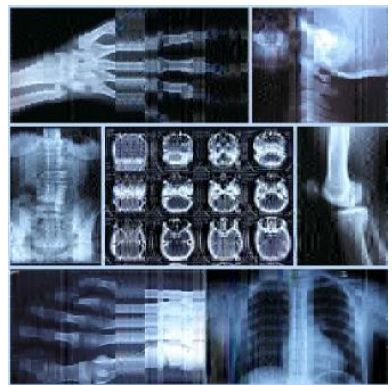

Codebook Size: 128

PNSR: 21.42dB; MAE: 6.40;

MSE: 468.14; CR: 99.86\%;

Abdomen- (I7)

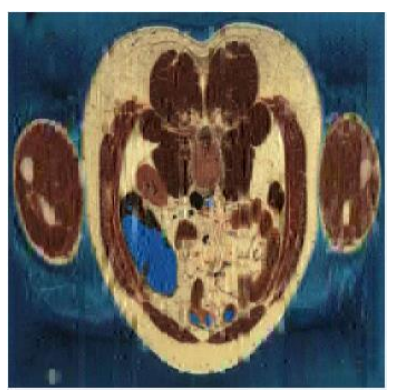

Codebook Size: 128

PNSR: 24.91dB; MAE: 4.47;

MSE: 209.49; CR: 99.87\%;

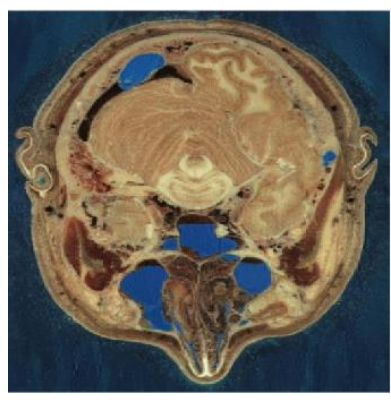

Codebook Size: 256

PNSR: 37.05dB; MAE: 1.19;

MSE: 12.81; CR: 99.82\%;

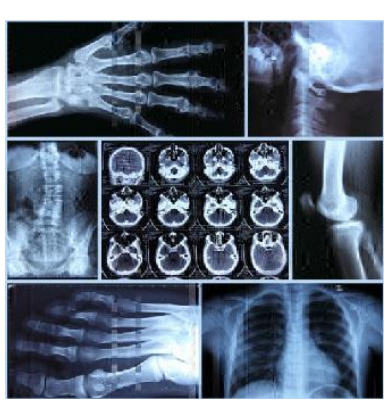

Codebook Size: 256

PNSR: 27.73dB; MAE: 2.83;

MSE: 109.54; CR: 99.82\%;

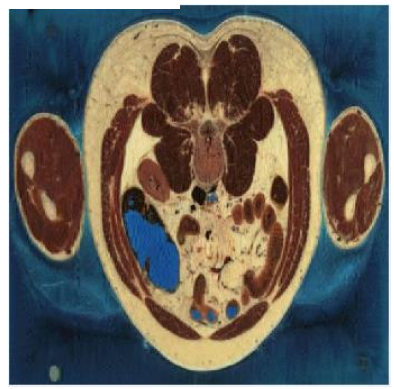

Codebook Size: 256

PNSR: 33.28dB; MAE: 1.80;

MSE: 30.54; CR: 99.82\%;

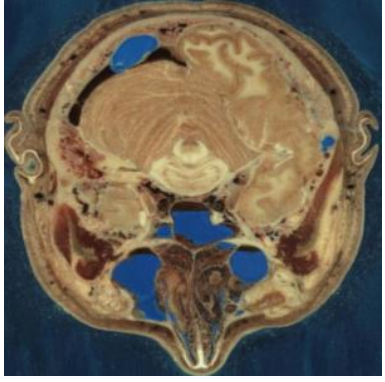

Codebook Size: 512

PNSR: Inf; MAE: 0;

MSE: 0; CR: 99.78\%;

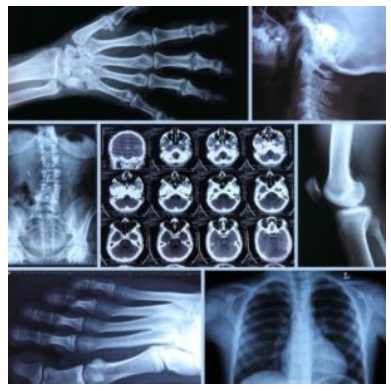

Codebook Size: 512

PNSR: Inf; MAE: 0;

MSE: 0; CR: 99.78\%;

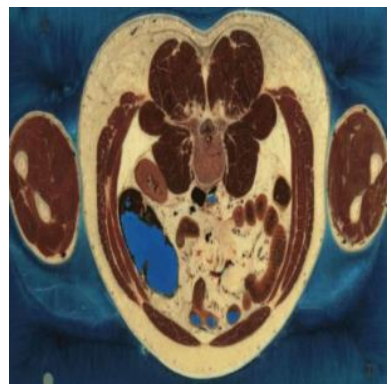

Codebook Size: 512

PNSR: Inf; MAE: 0;

MSE: 0; CR: 99.78\%;

Fig.3. Head, General and abdomen images compressed and decompressed using DChT 
Head- (I3)

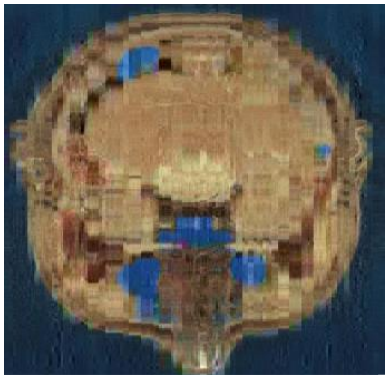

Codebook Size: 64

PNSR: 23.06dB; MAE: 6.07;

MSE: 321.0; CR: 99.90\%;

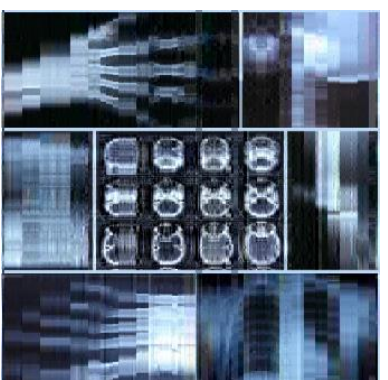

Codebook Size: 64

PNSR: 19.21dB; MAE: 9.09;

MSE: 780.1; CR: 99.89\%;

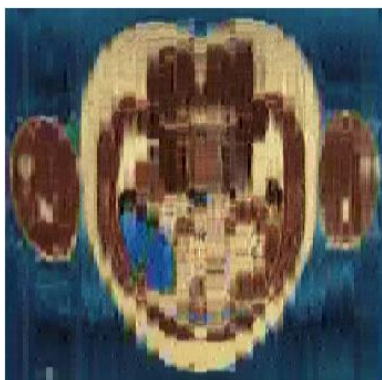

Codebook Size: 64

PNSR: 21.11dB; MAE: 7.30;

MSE: 502.9; CR: 99.89\%;

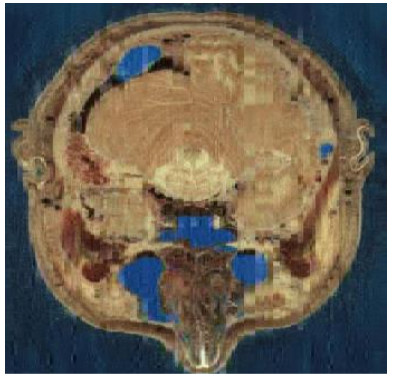

Codebook Size: 128

PNSR: 26.33dB; MAE: 4.02;

MSE: 151.29; CR: 99.86\%;

General- (I4)

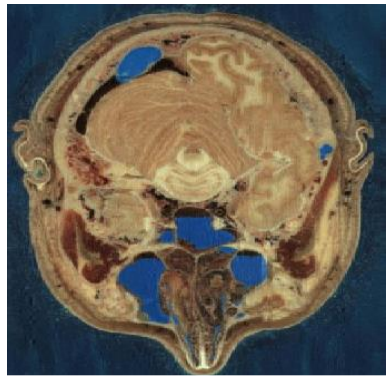

Codebook Size: 256

PNSR: 33.52dB; MAE: 1.76;

MSE: 28.89; CR: 99.82\%;

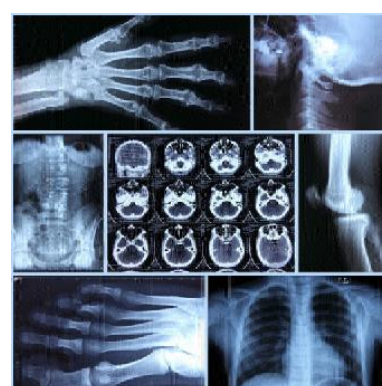

Codebook Size: 256

PNSR: 27.57dB; MAE: 2.83;

MSE: 109.54 ; CR: $99.82 \%$;

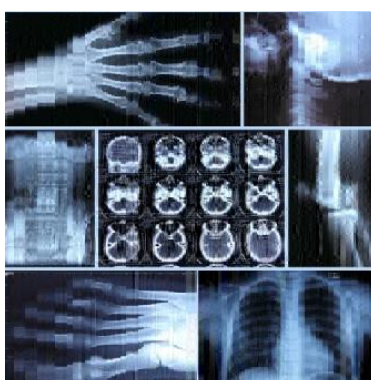

Codebook Size: 128

PNSR: 22.86dB; MAE: 5.50;

MSE: 336.2; CR: 99.85\%;

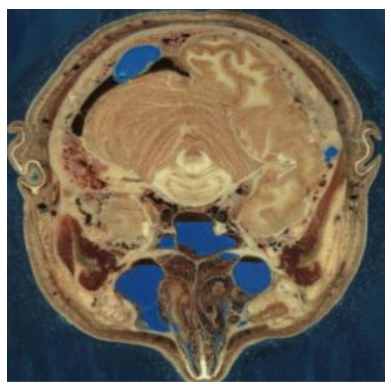

Codebook Size: 512

PNSR: Inf; MAE: 0;

MSE: 0; CR: 99.78\%;

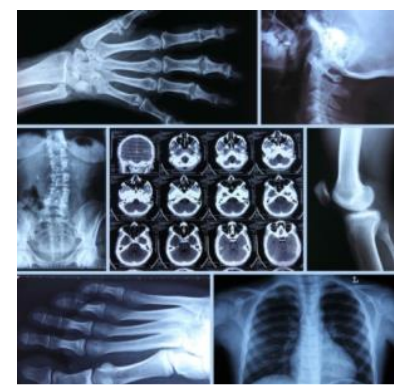

Codebook Size: 512

PNSR: Inf; MAE: 0;

MSE: 0; CR: 99.78\%;

Abdomen- (I7)

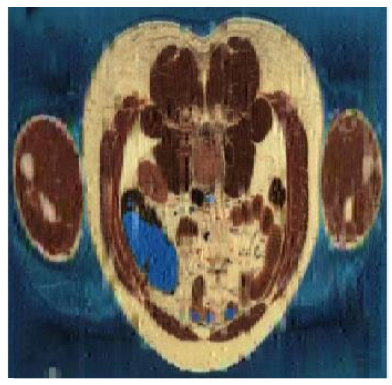

Codebook Size: 128

PNSR: 24.91dB; MAE: 4.47;

MSE: 209.49; CR: 99.87\%;

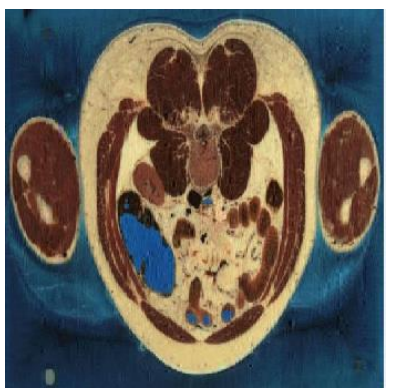

Codebook Size: 256

PNSR: 33.28dB; MAE: 1.80;

MSE: 30.54; CR: $99.82 \%$

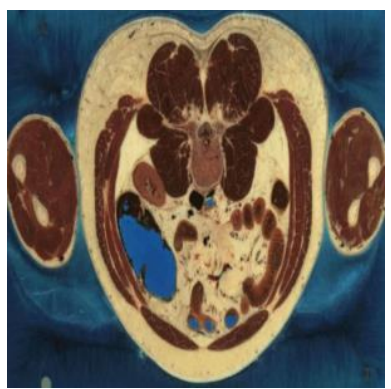

Codebook Size: 512

PNSR: Inf; MAE: 0;

MSE: 0; CR: 99.78\%;

Fig.4. Head, General and abdomen images compressed and decompressed using DWaT

Regarding the Fig.3 and Fig.4, we can say that using a subjective evaluation as Human Vision System (HVS), they are not the difference between original and decompressed image for a codebook size equal to 256 . Indeed for this codebook size, we obtain an average PNSR equal to 32, MAE equal to 2 and, it becomes difficult to see the difference between these images.

\section{B. Discussion}

It is easy to compare the performances of DChT and DWaT when analyzing data found in table 1 and in table
2. The compression and decompression time is greater when using the DChT transform than the DWaT transform. As an example for Head image, the compression time is $198.76 \mathrm{~s}$ for DChT and $46.15 \mathrm{~s}$ for DWaT when codebook size is 512. On the order hand, the results obtained with the DChT in terms of performances via the MSE, MAE and PSNR parameters are slightly higher than those of DWaT compression. In term of compression rate, the performance of DChT and DWaT are almost identical whatever the image or codebook size. 
Fig. 5.a compares MSE according to the codebook size obtained in DWaT and DChT. It can be observed that error decreases gradually with increase in codebook size. DWaT gives a maximum error. When codebook size is 256, average MSE is 32.001 in DChT and 50.165 in DWaT. Fig. 5.b shows MAE versus codebook size obtained in DWaT and DChT. Here also DWaT gives maximum error. MAE nearest to zero indicates image quality is closest to the original image. Fig. 6.a compares PSNR obtained in DWaT and DChT with different codebook size. DChT gives maximum PSNR. Average PSNR is $35.78 \mathrm{~dB}$ in DChT and $33.107 \mathrm{~dB}$ in DWaT when codebook size is 256. As in the Fig. 5, when codebook size increases less error occurs and hence PSNR increases indicating better image quality. Fig. 6.b shows CR versus codebook size obtained in DWaT and DChT. Here, DChT and DWaT give almost the same values between $99.78 \%$ and $99.90 \%$. Fig. 7.a and fig. 7.b show CT and DT obtained in DWaT and DChT respectively with different codebook size. Drastic difference is observed between DWaT and DChT. The fig. 8 compares against PNSR, MSE, MAE and CR obtained in DWaT and DChT with codebook size 256.

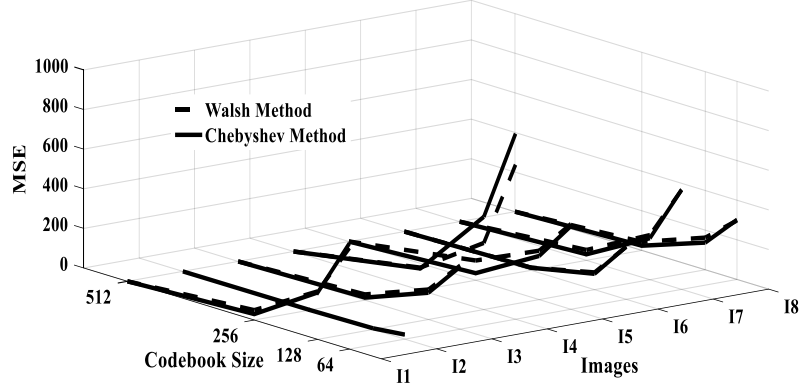

(a)

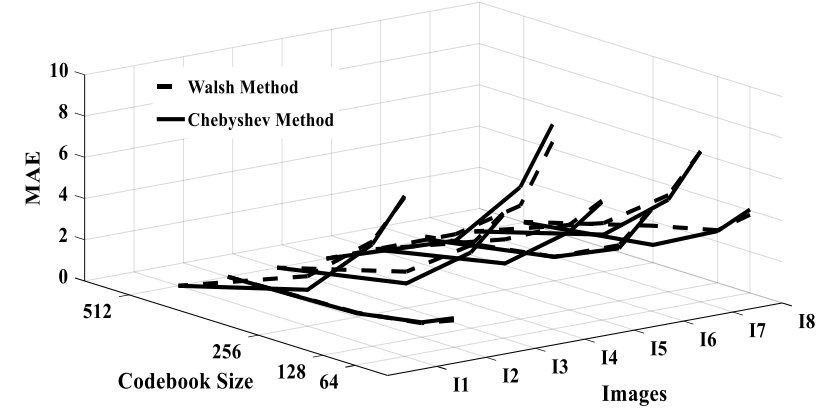

(b)

Fig.5. Three dimensions graphical representation of compression/ decompression using Walsh and Chebyshev methods. (a) MSE based on codebook size, (b) MAE based on codebook size.

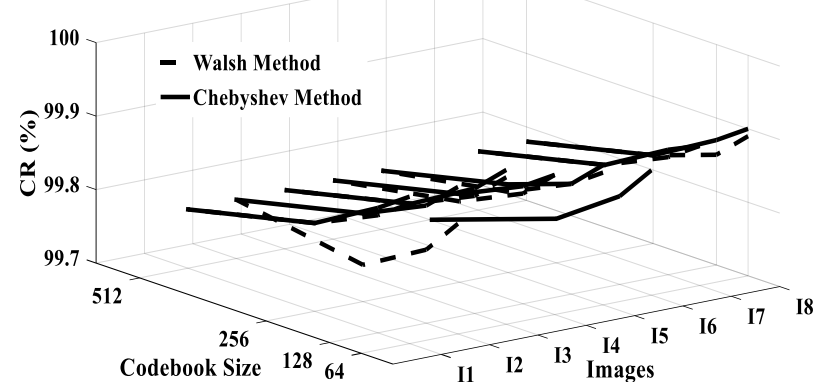

(a)

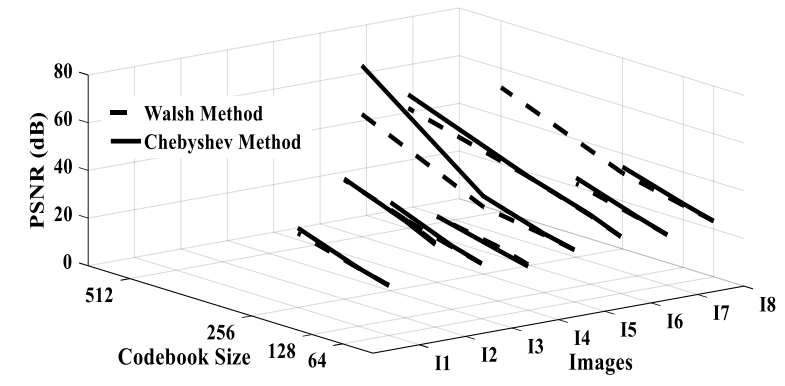

(b)

Fig.6. Three dimensions graphical representation of compression/ decompression using Walsh and Chebyshev methods. (a) CR based on codebook size, (b) PSNR based on codebook size

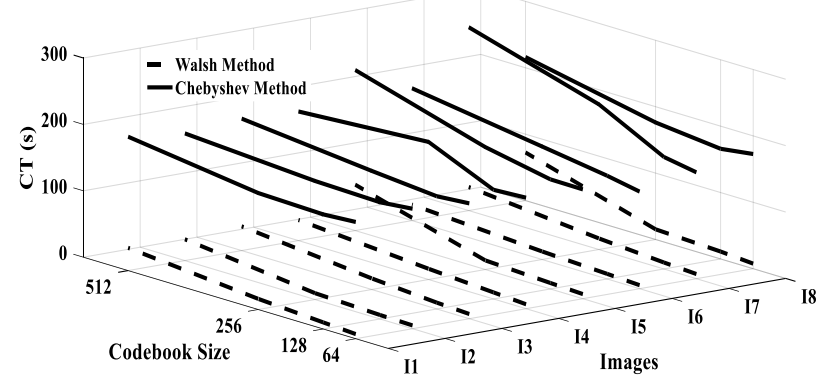

(a)

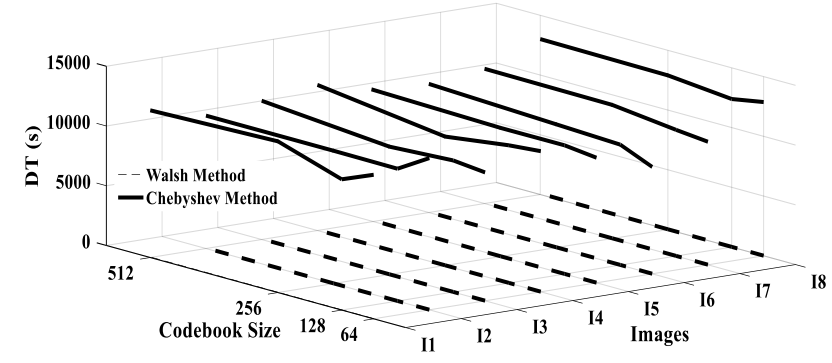

(b)

Fig.7. Three dimensions graphical representation of compression/ decompression using Walsh and Chebyshev methods. (a) CT based on codebook size, (b) DT based on codebook size.

Finally, to see a real difference between compression by DWaT and by DChT, we have represented a bar chart. These charts are represented in Fig.8. In these charts, we have represented the difference between the eight tests images and two methods (DwaT and DChT) for a codebook size equal to 256. Thus, we find that on the chart representing the PSNR, the DChT and DWaT are slightly different and, on that of the CR, the two methods 
(DwaT and DChT) are almost identical. On the charts of the MSE and the MAE we see a difference between our two methods and conclude that the DChT gives good parameters.

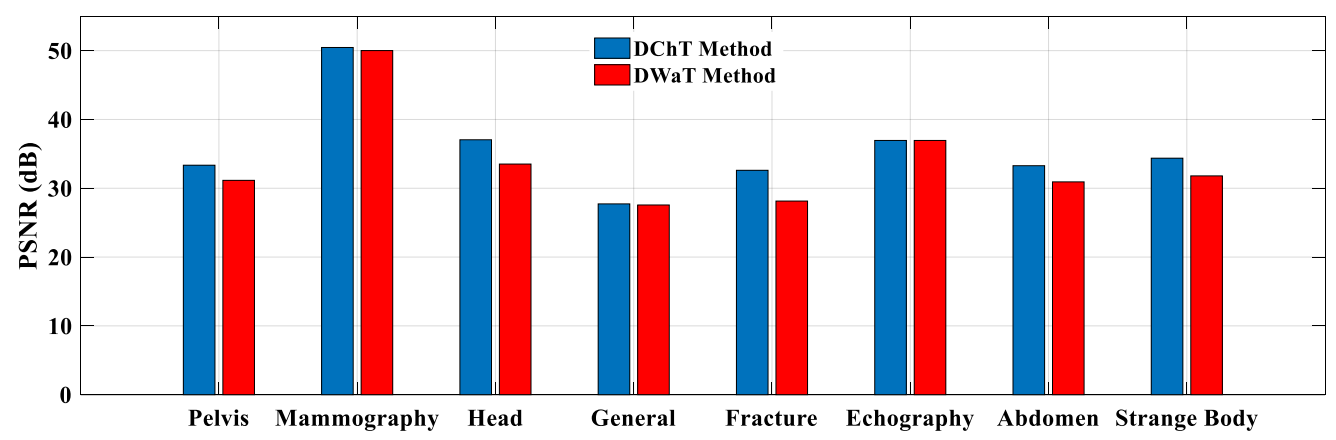

(a)

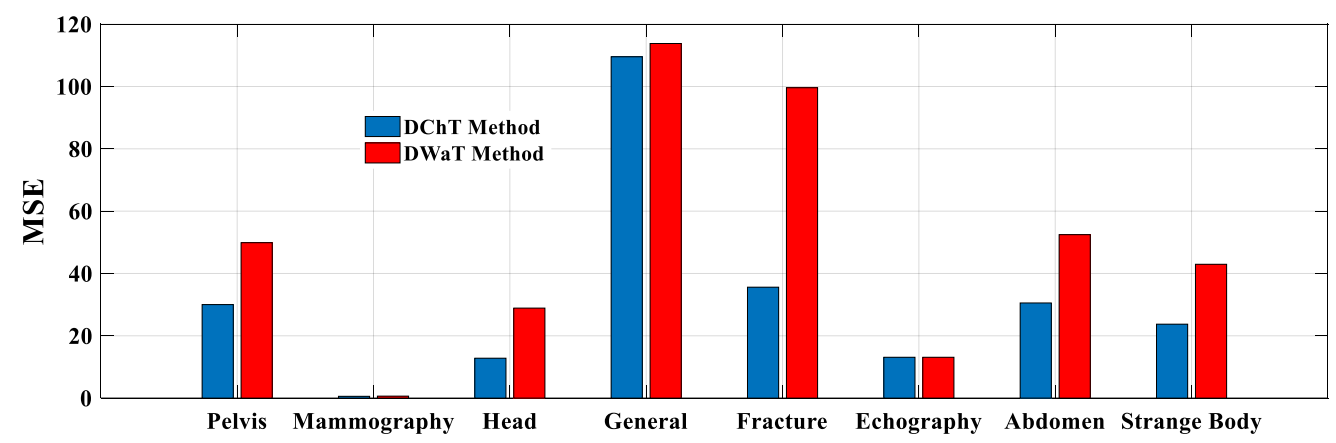

(b)

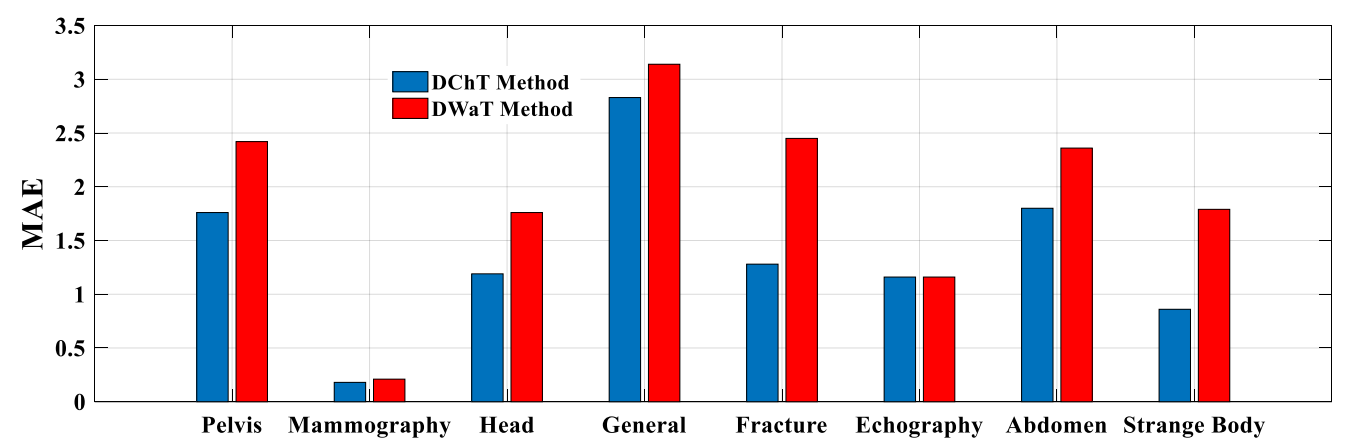

(c)

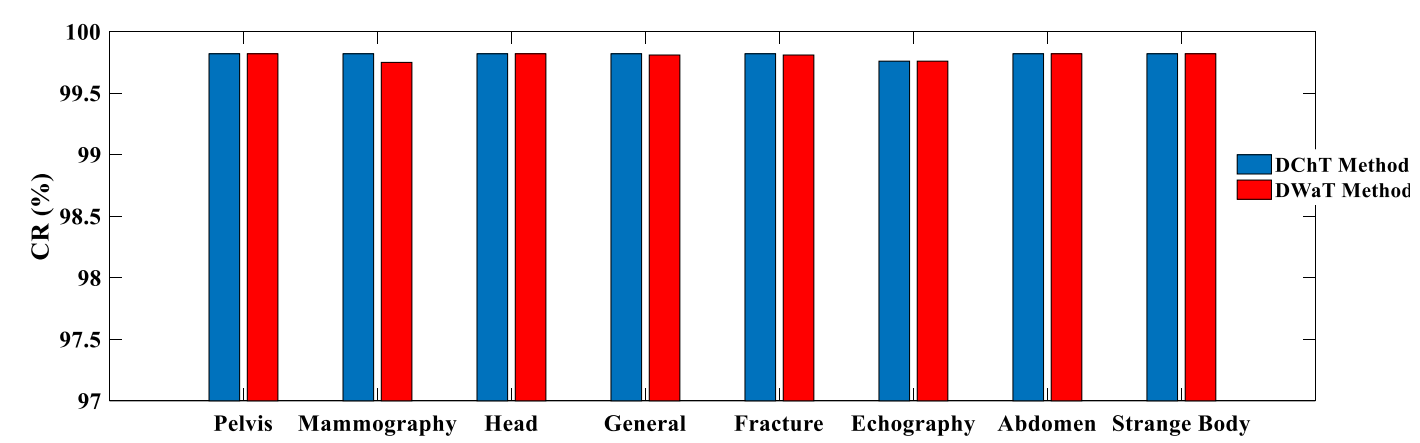

(d)

Fig.8. Parameters evaluation versus test images. (a): PSNR; (b): MSE; (c): MAE; (d): CR

In this work, we do not just simply use $K$-Means Clustering Algorithm to cluster the group of images and minimum Euclidean distance as in [24] or use LBG algorithm for vector quantization like in many works. We used the $K$-Means algorithm with the splitting method to optimize our codebook. Our Work is very adapted to the biomedical image because we can reconstruct an image without loss of information. 
Now, we compared our compression method with two recent work. Ayoobkhan el al in [59] introduce a lossy compression method. The method in [59] denoted PEVQ method uses the concept of vector quantization and prediction error. An optimal codebook is generated for VQ using a combination of two intelligent algorithms (artificial bee colony and genetic algorithms). Table 3 compares PSNR and CR obtained in introduce method (DWaT, DChT) and Ayoobkhan and al Method (PE-VQ) with codebook size 256 and 512. It can be observed that
PSNR increases gradually with an increase in codebook size. DWaT and DChT give maximum PSNR and equal to infinity when codebook size is 512 . PSNR equal to infinity indicates image quality remains the same with the original image. The difference is observed between $\mathrm{CR}$ in our method and method in [59]. For example, average CR is 99:1 in our method and 80:1 in [59] with codebook size is 256 . Our method outperforms and gives a good compromise between CR and PSNR. Fig. 9 shows the original image used for the comparison.

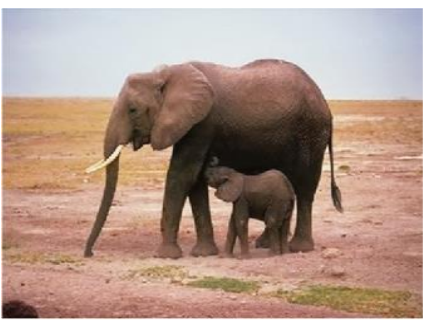

(a)

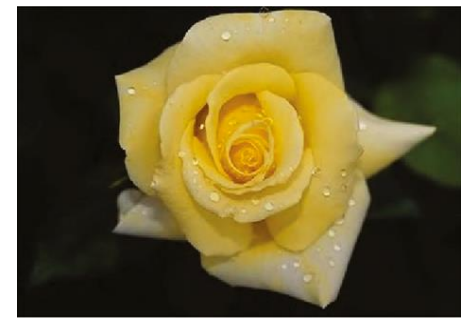

(b)

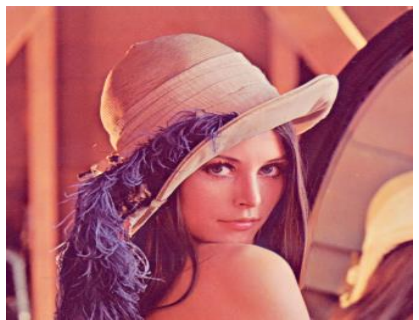

(c)

Fig.9 (a) Elephant; (b) Flower; (c) Lena [19, 59]

Table 3. Performance comparison (PSNR and CR) of the introduced method and [59] Method.

\begin{tabular}{|c|c|c|c|c|c|c|c|c|c|c|c|c|}
\hline \multirow{3}{*}{$\begin{array}{c}\text { Method } \\
\text { Codebook size }\end{array}$} & \multicolumn{8}{|c|}{ Our Method } & \multirow{2}{*}{\multicolumn{4}{|c|}{$\begin{array}{c}\text { Ayoobkhan and al Method [58] } \\
\text { PE-VQ }\end{array}$}} \\
\hline & \multicolumn{4}{|c|}{ DWaT } & \multicolumn{4}{|c|}{ DChT } & & & & \\
\hline & \multicolumn{2}{|c|}{256} & \multicolumn{2}{|c|}{512} & \multicolumn{2}{|c|}{256} & \multicolumn{2}{|c|}{512} & \multicolumn{2}{|c|}{256} & \multicolumn{2}{|c|}{512} \\
\hline Parameters & $\begin{array}{c}\text { PSNR } \\
\text { (dB) }\end{array}$ & CR & $\begin{array}{c}\text { PSNR } \\
(\mathrm{dB})\end{array}$ & $\mathrm{CR}$ & $\begin{array}{c}\text { PSNR } \\
(\mathrm{dB})\end{array}$ & CR & $\begin{array}{c}\text { PSNR } \\
(\mathrm{dB})\end{array}$ & CR & $\begin{array}{c}\text { PSNR } \\
(\mathrm{dB})\end{array}$ & CR & $\begin{array}{c}\text { PSNR } \\
(\mathrm{dB})\end{array}$ & $\mathrm{CR}$ \\
\hline Elephant & 35.43 & $99: 1$ & Inf & $99: 1$ & 38.92 & $99: 1$ & Inf & $99: 1$ & 39.04 & $80: 1$ & 42.5 & $73: 1$ \\
\hline Flower & 42.92 & $99: 1$ & Inf & 99:1 & 45.13 & 99:1 & Inf & $99: 1$ & 38.91 & $80: 1$ & 42.5 & $73: 1$ \\
\hline
\end{tabular}

H. B. Kekre, and al in [19] used some parameters (MAE, Compression Ratio and AFCPV) to evaluate their algorithm. Table 4 gives the values of MAE and CR according to codebook size on the Lena color image. Table 4 compares CR and MAE obtained in our method and Kekre et al. method according to codebook size. Using KFCG and KMCG less error is obtained than an error in DWaT and DChT when the codebook size is 16.
MAE 8.36 and 8.34 is obtained for DChT and KFCG respectively when the codebook size is 32 . MAE nearest to zero indicates image quality is closest to the original image. CR in DWaT and DChT is higher than in KFCG and KMCG. We obtained an average $99.90 \%$ and $99.34 \%$ in our method and Kekre and al method respectively for a codebook size 32. DChT outperforms DWaT and Kekre et al. method.

Table 4. Performance comparison (MAE and CR) of the introduced method and Method proposed in [19].

\begin{tabular}{|c|c|c|c|c|c|}
\hline \multirow{2}{*}{ Codebook size } & \multirow{2}{*}{ Parameters } & \multicolumn{2}{|c|}{ Our Method } & \multicolumn{2}{c|}{ H. B. Kekre, and al Method [19] } \\
\cline { 3 - 6 } & & DWaT & DChT & KMCG+HWT & KFCG+HWT \\
\hline \multirow{2}{*}{16} & MAE & 10.12 & 10.07 & 10.09 & 9.32 \\
\cline { 2 - 6 } & CR (\%) & 99.92 & 99.92 & 99.47 & 99.47 \\
\hline \multirow{2}{*}{32} & MAE & 8.31 & 8.28 & 8.89 & 9.34 \\
\cline { 2 - 6 } & CR (\%) & 99.91 & 99.90 & 99.34 & - \\
\hline \multirow{2}{*}{64} & MAE & 5.51 & 5.44 & - & - \\
\cline { 2 - 5 } & CR $(\%)$ & 99.87 & 99.89 & - & -34 \\
\hline
\end{tabular}

Thus in both comparison, our method has given the best result about related work. We can conclude that when the image is transformed with DChT or DWaT, and the codebook is calculated with a ML algorithm $(K$ Means and splitting method), the compression is performed with very good parameters.

\section{CONCLUSION}

Our aim in this paper was to present a new scheme for biomedical color image compression. We present an optimal method for biomedical images compressing using orthogonal transform (DWaT and DChT) and 
vector quantization based on ML algorithm. ML algorithm ( $K$-Means and splitting method) used to calculate and generate the codebook. VQ is applied on transform domain images. Using this scheme, image quality is obtained at an average CR $99.85 \%$. MAE gives clear idea of image quality. At codebook size 256, an average MAE 1.38 and 1.89 is given for DChT and DWaT respectively. DChT outperforms DWaT in terms of PSNR, MSE and MAE. Computing time of DChT is very large compared to DWaT. As compared to Kekre method in [19] and Ayoobkhan method in [59] according to codebook size, DWaT gives less error and DChT gives least error among all with better image quality. The comparison demonstrates the effectiveness of the proposed scheme in biomedical color image compression. An extension to this work is to build a big data environment in order to simulate our proposed compression algorithm.

\section{REFERENCES}

[1] Bruylants T, Munteanu A, Schelkens P, "Wavelet Based Volumetric Medical Image Compression", Elsevier Signal Processing: Image Communication. Vol.31, pp. 112-133, 2015.

[2] Sayood K, Introduction to Data Compression, third ed., Morgan Kaufmann, San Francisco, 2006.

[3] S. K. Singh and S. Kumar, "Mathematical Transforms and Image Compression: A review", Maejo. Int. J. Sci. Technol pp. 235-249, 2010.

[4] Salomon David, A Concise Introduction to data compression, Springer-Verlag, London, 2008.

[5] Farelle P, Recursive Block Coding for Image data compression, Springer-Verlag, New York, 1990.

[6] S. Lee, "Compression image reproduction based on block decomposition", IET Image Processing. Vol.3, No.5, pp. 188-199, 2009.

[7] R. Starosolski, "New simple and efficient color space transformations for lossless image Compression", Elsevier J. Vis. Commun. Image R. Vol.25 pp. 1056-1063, 2014.

[8] Dayanand G. Savakar and Shivanand Pujar ," Digital Image Watermarking Using DWT and FWHT ", International Journal of Image, Graphics and Signal Processing(IJIGSP), Vol.10, No.6, pp. 50-67, 2018. DOI: 10.5815/ijigsp.2018.06.06

[9] Taubman David, Marcellin M, JPEG2000 Image Compression fundamentals, Standards and Practice, Kluwer Academic Publishers, Boston, 2002.

[10] R. Mukundan, "Transform Coding Using Discrete Tchebichef Polynomials", Proceedings IASTED International Conference on Visualization Imaging and Image Processing, pp. 270-275, 2006.

[11] Azman Abu and Sahib S, "Spectral Test via Discrete Tchebichef Transform for Randomness", International Journal of Cryptology Research, Vol. 3, pp.1-14, 2011.

[12] Prattipati S, Swamy M, Meher P, "A Comparison of Integer Cosine and Tchebychev Transforms for Image Compression Using Variable Quantization", Journal of Signal and Information Processing, Vol. 6, pp. 203-216, 2015.

[13] Senapati R, Pati U, Mahapatra K, "Reduced memory, low complexity embedded image compression algorithm using hierarchical listless discrete Tchebichef transform", IET Image Processing, Vol. 8, No.4, pp. 213-238, 2014.
[14] Pan Z, Kotani K and Ohmi T, "Fast Encoding Method for Image Vector Quantization by Using Partial Sum Concept in Walsh Domain”, IEEE Signal Processing Conference, $4 \mathrm{p}, 2005$.

[15] Lu T, Chang C, A Survey of VQ Codebook Generation, Journal of Information Hiding and Multimedia Signal Processing, Vol.1, pp. 190-203, 2010.

[16] GERSHO A, "On the Structure of Vector Quantizers", IEEE Trans. on Inform. Theory, Vol. 28, 1982.

[17] GRAY M, "Vector Quantization", IEEE ASSP Magazine, pp. 4-29, 1984.

[18] Panchanathan S, Goldberg M, "Adaptive Algorithms for Image Coding Using Vector Quantization", Elsevier Signal Processing: Image Communication, Vol. 4, pp. 8192, 1991.

[19] Kekre H, Natu $P$ and Sarode T, "Color Image Compression using Vector Quantization and Hybrid Wavelet Transform", Elsevier Procedia Computer Science, Vol. 89, pp. 778-784, 2016.

[20] Zhong S, Chin F, Yun Shi Q, "Adaptive hierarchical vector quantization for image coding: new results", Optical Engineering, Vol.34, pp. 2912-2917, 1995.

[21] Chuang J, Hu Y, "An adaptive image authentication scheme for vector quantization compressed image", Elsevier J. Vis. Commun. Image R, Vol. 22, pp. 440-449, 2011.

[22] Comaniciu D, Grisel R, "Image Coding Using Transform Vector Quantization with Training Set Synthesis", Elsevier Signal Processing, Vol. 89, pp. 1649-1663, 2002.

[23] Setiawan A, Suksmono A and Mengko T, "Color Medical Image Vector Quantization Coding Using K-Means: Retinal Image", Springer IFMBE Proceedings, Vol. 23, pp. 911-914, 2009.

[24] Choudhry M and Kapoor R, "Performance Analysis of Fuzzy C-Means Clustering Methods for MRI Image Segmentation", Elsevier Procedia Computer Science, Vol. 89, pp. 749-758, 2016

[25] Morales-Mendoza L, Gamboa-Rosales H, Shmaliy Y, “A new class of discrete orthogonal polynomials for blind fitting of finite data", Elsevier Signal Processing, Vol. 93, 1785-1793, 2013.

[26] Shakibaei Asli B, Paramesran E, Lim C, "The fast recursive computation of Tchebichef moment ant its inverse transform based on Z-transform", Elsevier Digital Signal Processing, Vol.23, No.5, pp.1738-1746, 2013.

[27] Nakagaki K and Mukundan R, "A Fast 4x4 Forward Discrete Tchebichef Transform Algorithm", IEEE Signal Processing Letters Vol. 14, No.10, pp. 684-687, 2007.

[28] Ernawan F and Azman Abu N, "Efficient Discrete Tchebichef on Spectrum Analysis of Speech Recognition", International Journal of Machine Learning and Computing, Vol.1, pp. 1-6, 2011.

[29] Xiao B, Lu G, Zhang Y, Li W, Wang G, "Lossless image compression based on integer Discrete Tchebichef Transform", Elsevier Neurocomputing, Vol. 214, pp. 587593, 2016.

[30] Gegum A, Manimegali D, Abudhahir A, Baskar S, "Evolutionary optimized discrete Tchebichef moments for image compression applications, Turk J Elec Eng \& Comp Sci, Vol.24“ pp. 3321-3334, 2016. doi:10.3906/elk-1403-318.

[31] Abu N, Wong S, Rahmalan H, Sahib S, "Fast and Efficient 4x4 Tchebichef Moment Image Compression", Majlesi Journal of Electrical Engineering, Vol.4, pp. 1-9, 2010.

[32] Chirikjian G and Kyatkin A0233ngineering Applications of Noncommutative Harmonic Analysis: With Emphasis 
on Rotation and Motion Groups, CRC Press LLC, Boca Raton, Florida, 2000. ISBN: 0-8493-0748-1

[33] Aloui N, Bousselmi S, Cherif A., "Speech Compression Based on Discrete Walsh Hadamard Transform", Int.J. Information Engineering and Electronic Business, Vol.3, pp. 59-65, 2013.

[34] Karagodin M, Polytech T, Russia U, Osokin A, "Image Compression by Means of Walsh Transform", IEEE Modern Technique and Technologies, pp. 173-175, 2002.

[35] Linde Y, Buzo A, Gray M, "An Algorithm for Vector Quantizer Design", IEEE Transactions on Communications, Vol. 28, No. 1, pp. 84- 95, 1980.

[36] Le Bail and Mitiche A, "Vector Quantization of Images Using Kohonen Neural Network", Elsevier Signal Processing, Vol. 6, pp. 529-539, 1989.

[37] Huang B, Wang Y and Chen J, "ECG Compression Using the Context Modeling Arithmetic Coding with Dynamic Learning Vector-Scalar Quantization", Elsevier Biomedical Signal Processing and Control, Vol. 8, No. 1, pp. 59-65, 2013.

[38] Wang $X$ and Meng J, "A 2-D ECG Compression Algorithm Based on Wavelet Transform and Vector Quantization", Elsevier Digital Signal Processing, Vol.18, No.2, pp. 179-188, 2008.

[39] Cosman P, Tseng C, Gray M, Olshen R, et al., "TreeStructured Vector Quantization of CT Chest Scans: Image Quality and Diangnostic Accuracy", IEEE Tansactions on Medical Image, Vol.12, No. 4, pp. 727-739, 1993.

[40] De A and Guo, "An adaptive vector quantization approach for image segmentation based on SOM network", Elsevier Neurocomputing, Vol. 149, pp. 48-58, 2015.

[41] Laskaris N, Fotopoulos S, "A novel training scheme for neural-network based vector quantizers and its application in image compression", Elsevier Neurocomputing Vol. 61, pp. 421-427, 2004

[42] Nagaradjane $P$, Swaminathan $S$, Krishnan $S$, "Performance of space-division multiple-access system using preprocessing based on feedback of vectorquantized channel spatial information", Elsevier Computers and Electrical Engineering, Vol.40, pp. 13161326 ,

2014. http://dx.doi.org/10.1016/j.compeleceng.2014.01.002.

[43] Cosman P, Gray R and Vetterli M, "Vector Quantization of Image Subbands: A Survey", IEEE Tansactions on Image Processiong, Vol.5, No.3, pp. 202-224, 1996.

[44] Choudhry M and Kapoor R, "Performance Analysis of Fuzzy C-Means Clustering Methods for MRI Image Segmentation", Elsevier Procedia Computer Science, Vol. 89, pp. 749-758, 2016.

[45] Naldi M, Campello R, "Comparison of distributed evolutionary $k$-means clustering algorithms", Elsevier Neurocomputing, Vol. 163, pp. 78-93, 2015.

[46] Dhanachandra N, Manglem K and Jina Chanu Y, "Image Segmentation using K-means Clustering Algorithm and Subtractive Clustering Algorithm", Elsevier Procedia Computer Science, Vol.54, pp. 764 - 771, 2015.

[47] Cuomo S, De Angelis V, Farina G, Marcellino L, Toraldo G, "A GPU-accelerated parallel K-means algorithm", Elsevier Computers and Electrical Engineering, $\quad$ pp.1-13., 2017, https://doi.org/10.1016/j.compeleceng.2017.12.002

[48] Lakhdar A, Khelifi M, Beladgham M, Aissa M, Bassou A, "Image Vector Quantization Codec Indexes Filtering", Serbian Journal of Electrical Engineering, Vol.9, 263277, 2012.
[49] Antonini M, Barlaud M, Mathieu P, Optimal "Codebook and New Strategy of Image vector Quantization", Twelfth colloquium Gretsi, Juan les Pins, 4p, 1989.

[50] Franti P, Kaukoranta T, Nevalainen O, "On the Splitting Method for VQ Codebook Generation", Optical Engineering, Vol. 36, No.11, pp. 3043-3051, 1997.

[51] Huffman David, "A Method for the Construction of Minimum-Redundancy Codes", Proceeding of the I.R.E, Vol.40, pp. 1098-1101, 1952.

[52] Sohag Kabir,"A Compressed Representation of MidCrack Code with Huffman Code", I.J. Image, Graphics and Signal Processing (IJIGSP), vol.7, no.10, pp.11-18, 2015.DOI: 10.5815/ijigsp.2015.10.02

[53] Berman P, Karpinski M, Nekrich Y, "Approximating Huffman Codes in Parallel", Elsevier Journal of Discrete Algorithms, Vol.5, No.3, pp. 479-490, 2007.

[54] Tomasz Biskup $M$ and Plandowski W, "Shortest Synchronizing Strings for Huffman Codes", Elsevier Theoretical Computer Science, Vol.410, No. 30-40, pp. 3925-3941, 2009.

[55] S. Shunmugan, P. Arockia Jansi Rani, "Secured Lossy Color Image Compression Using Permutation and Predictions", International Journal of Image, Graphics and Signal Processing (IJIGSP), Vol.9, No.6, pp.29-36, 2017.DOI: 10.5815/ijigsp.2017.06.04

[56] Ananthi V, Balasubramaniam P, "A new image denoising method using interval-valued intuitionistic fuzzy sets for the removal of impulse noise", Elsevier Signal Processing Vol.121 pp. 81-93, 2015.

[57] http://www.atlas-imagerie.fr/, (accessed 10.04.17).

[58] 'US National Library of Medicine', https://www.nlm.nih.gov/, (accessed 12.04.17).

[59] Ayoobkhan M, Chikkannan E and Ramakrishnan K, "Lossy image compression based on prediction error and vector quantisation", Springer EURASIP Journal on Image and Video Processing, Vol. 35, pp. 1-13, 2017.

\section{Authors' Profiles}

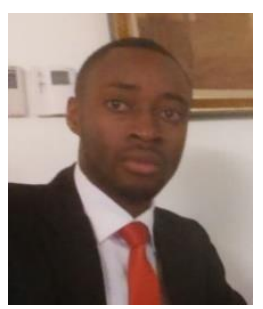

Tchagna Kouanou Aurelle was born in 1990 in Dschang-Cameroon. He obtained a MS degree in Electronic in 2013 from University of Dschang, he graduated in Telecommunication engineering from the National Advanced School of Posts, Telecommunications and Information Communication Technologies (SUP' PTIC) Yaounde-Cameroon in 2015. Actually, Mr Tchagna perform a $\mathrm{PhD}$ degree at University of Dschang where his main items of research include Biomedical image processing, Artificial Intelligence, Big Data, Deep Learning, Machine learning and Internet of Things.

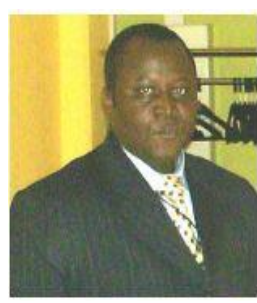

Tchiotsop Daniel was born in 1965 in Tombel - Cameroon. He graduated in Electromechanical engineering from the Ecole Nationale Supérieure Polytechnique (ENSP) of YaoundéCameroon in 1990, he obtained a MS degree in Solid Physics in 1992 from the Faculty of Science of the University of Yaoundé I, a MS degree in Electrical 
Engineering and Telecommunication in 2003 from ENSPYaoundé and a PHD at INPL (Institut National Polytechnique de Lorraine), Nancy-France, in 2007. Dr TCHIOTSOP teaches in the Department of Electrical Engineering of the FOTSO Victor University Institute of Technology - University of Dschang since 1999 where he is actually assistant Professor and the Head of Department. $\mathrm{He}$ is with the Laboratoire d'Automatique et d'Informatique Appliquée (LAIA) where his main items of research include Biomedical Engineering, Biomedical signal and image processing, Telemedicine and intelligent systems.

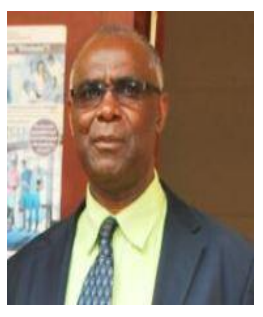

Pr. Tchinda Rene teaches in the Department of Thermal Science, Energy and Environmental Engineering of the FOTSO Victor University Institute of Technology - University of Dschang where he is actually the deputy Director. $\mathrm{He}$ is with the Laboratoire d'Ingénierie des Systèmes Industriels et de l'Environnement (LISIE).

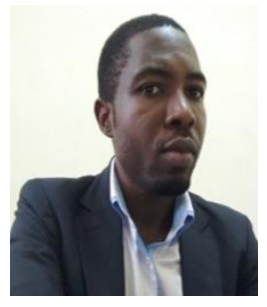

Tchapga Tchito Christian was born in 1981 in Yaoundé-Cameroon. $\mathrm{He}$ obtained a MSc degree in Electronics in 2010 from University of Dschang, $\mathrm{Mr}$ Tchapga is attending a $\mathrm{PhD}$ program in Electronics in University of Dschang where his main items of research include Biomedical Signal Processing, Artificial Intelligence, and Internet of Things, Telemedecine and Biomedical device design. He teaches in the Department of Electrical and Electronic Engineering of the College of Technology / University of BUEA, since 2012 where he is actually the Head of Division of Internship. He works with, Laboratoire d'Electronique et Traitement du Signal (LETS), Laboratoire d'Automatique et d'Informatique Appliquée (LAIA), Research Group on Experimental and Applied Physics for Sustainable Development (EAPHYSUD) where his main items of research include Web Technologies, Embedded Systems, Computational Intelligence.

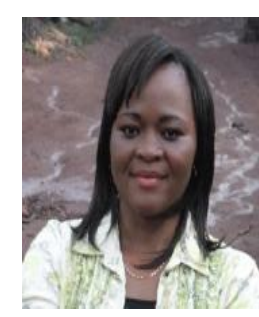

Kengnou Telem Adelaide Nicole was born 1977 in Dschang - Cameroon. In 2003, she was graduated at the Advanced Teacher's Training College for Technical Education (ENSET) - University of Douala, with DIPET 1 (Bachelor in Electrical and Electronics Engineering). In 2005, she obtained the DIPET 2 at the same institution. She obtained the Master degree in Electronics in 2012 at the faculty of Science of the University of Dschang. She is currently a Technical High School teacher in electronics engineering. In parallel to her job, Mrs. KENGNOU has a $\mathrm{PhD}$ degree in Electronic from University of Dschang. Her research interests are telemedicine, secure transmission of physiological signals and images, wireless communication and image processing.

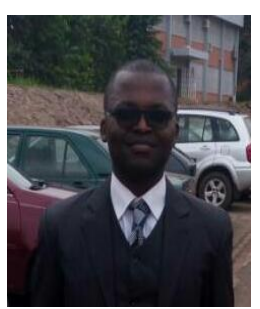

Kengne Romanic was born in 1983 in Ngaroundere-Cameroon. He obtained a MS degree in Electronic in 2010 from University of Dschang, he graduated in Telecommunication engineering from National Advanced School Polytechnics, of the University of Yaounde 1 (ENSPYaoundé) Cameroon in 2017. He performed now a $\mathrm{PhD}$ degree at University of Dschang where his main items of research include Dynamic of Electronic System and Fractionnal System, Chaotic System, Antenna System, Machine Learning and Big Data.

How to cite this paper: Aurelle Tchagna Kouanou, Daniel Tchiotsop, René Tchinda, Christian Tchito Tchapga, Adelaide Nicole Kengnou Telem, Romanic Kengne, "A Machine Learning Algorithm for Biomedical Images Compression Using Orthogonal Transforms", International Journal of Image, Graphics and Signal Processing(IJIGSP), Vol.10, No.11, pp. 38-53, 2018.DOI: 10.5815/ijigsp.2018.11.05 